\title{
236mW Average Second-Harmonic Power Generated from Periodically Poled Silica Fibres
}

\author{
Albert Canagasabey ${ }^{1}$, Costantino Corbari ${ }^{1}$, Flavien Liegeois ${ }^{2}$, Alexey V. Gladyshev ${ }^{3}$, Sebastien Guillemet ${ }^{2}$, \\ Yves Hernandez ${ }^{2}$, Mikhail V. Yashkov ${ }^{4}$, Alexey Kosolapov ${ }^{3}$, Evgeny M. Dianov ${ }^{3}$, Morten Ibsen ${ }^{1}$ and Peter \\ G. Kazansky ${ }^{1}$
}

\author{
1. Optoelectronics Research Centre, University of Southampton, SO17 1BJ, United Kingdom \\ 2. Applied Photonics Department, Multitel, 2 av Pierre et Marie Curie, Parc Initialis, 7000, Mons, Belgium \\ 3. Fiber Optics Research Center, Russian Academy of Sciences, 38 Vavilov Street, 119333 Moscow, Russia \\ 4. Institute of Chemistry of High-Purity Substances, 49 Tropinin St, 603950, Novgorod, Russia
}

Thermally poled silica fibres are an attractive all-fibre solution to frequency conversion of high power fibre lasers. In comparison to nonlinear crystals, they offer inherently lower insertion losses, higher optical damage threshold, greater stability and ruggedness intrinsic to all-fibre solutions. Moreover, the relatively low secondorder-nonlinearity $(0.1-0.2 \mathrm{pm} / \mathrm{V})$ can be compensated by extending the length of the periodically poled fibre.

In this work, the generation of as much as $236 \mathrm{~mW}$ single-mode second-harmonic (SH) light from a periodically poled fibre (PPSF) pumped by a high power fibre laser is demonstrated. The result shows the ability of the PPSF to handle high average pump powers $(\sim 1.6 \mathrm{~W})$ without any signs of decay of the second-order nonlinearity from day to day operation. The average power shows a 35-times improvement over the best results on PPSF to date and the corresponding average efficiency exceeding $15 \%$ was achieved with 20-times lower peak power [1]. The PPSF device was fabricated by the insertion of wire electrodes into the holes of a specialty twin-hole germanosilicate fibre (Figure 1(a) -inset). The device was subsequently poled at an optimum temperature of $210^{\circ} \mathrm{C}$ and the simultaneous application of $11 \mathrm{kV} \mathrm{DC}$ within the electrodes over a 1 hour period. A uniform $\chi^{(2)}$ of $\sim 0.12 \mathrm{pm} / \mathrm{V}$ was measured after poling. Quasi phase matching (QPM) for second-harmonicgeneration (SHG) was achieved through point-by-point periodic $(\Lambda=66.5 \mu \mathrm{m})$ UV erasure [2] at optimised conditions over a record length of $32 \mathrm{~cm}$. Splice losses to SMF28 fibre were measured to be $0.5 \mathrm{~dB}$ at $1550 \mathrm{~nm}$ and $1.0 \mathrm{~dB}$ at $780 \mathrm{~nm}$. The device was initially characterised using a $9 \mathrm{~mW}$ continuous-wave tunable external cavity diode laser. The acceptance bandwidth of the device was measured to be $0.46 \mathrm{~nm}$ in agreement with theoretical predictions. The almost perfect $\operatorname{sinc}^{2}$ profile of the tuning curve is a testament to the extremely good quality of the quasi-phase matched grating over the whole $32 \mathrm{~cm}$ length (Figure 1(a)). From this measurement, the normalised conversion efficiency was estimated to be $7.34 \times 10^{-2} \% / \mathrm{W}$, translating to a 3.3 -fold improvement in comparison to the previously best reported value [2] and 14-fold over [1].
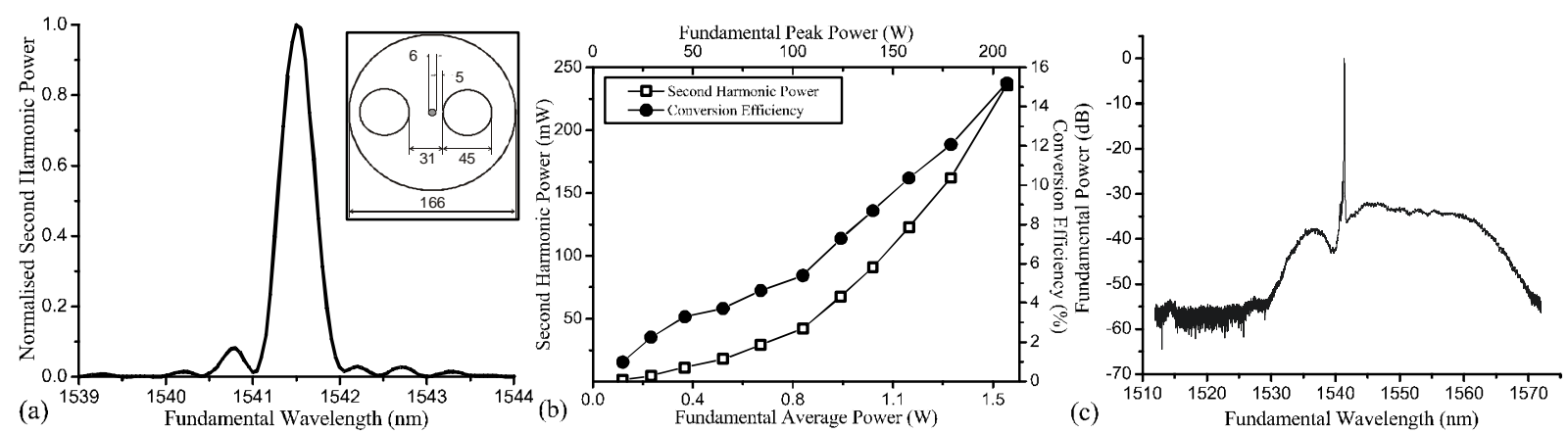

Figure 1 (a) Second-harmonic tuning curve of the PPSF device (inset) specialty twin-hole fibre, dimensions in $\mu$ m (b) Average second-harmonic power and average conversion efficiency (c) Er/Yb fibre laser source spectrum (RBW 0.01nm)

A high power tunable Er/Yb fibre laser MOPA source of $3 \mathrm{MHz}$ repetition rate and $2.5 \mathrm{~ns}$ pulse duration was employed to characterise the PPSF device. The quadratic dependence of the average SH power at the wavelength of $771.75 \mathrm{~nm}$ against the fundamental source power operating at $1541.5 \mathrm{~nm}$ is shown in Figure 1(b). The maximum average conversion efficiency was calculated to be $15.2 \%$ with just $207 \mathrm{~W}$ of fundamental peak power (Figure $1(\mathrm{~b})$ ). The obtained average $\mathrm{SH}$ power was $236 \mathrm{~mW}$ in a single-mode $\left(\mathrm{LP}_{01}\right)$. A further improvement of the conversion efficiency is theoretically possible by scaling to even longer lengths, through the enhancement of the effective nonlinearity or with higher peak power fibre laser sources [3].

\section{References}

1. V. Pruneri et al. "Greater than $20 \%$ efficient frequency doubling of $1532 \mathrm{~nm}$ pulses in quasi-phase-matched germanosilicate fibres," Optics Letters. 24, pp. 208-210, (1999).

2. C. Corbari et al. "All-fibre frequency conversion in long periodically poled silica fibres," in proceedings to OFC 2005, Anaheim, U.S.A., paper OFB3.

3. A. A. Fotiadi et al. "All-fiber frequency doubled Er/Brillouin laser", in proceedings to CLEO/QELS 2006, Long Beach, U.S.A., paper CTuI3. 